\title{
Cyanide Photochemistry and Nitrogen Fractionation in the Mwc 480 Disk
}

\section{Citation}

Guzmán, V. V., K. I. Öberg, R. Loomis, and C. Qi. 2015. "Cyanide Photochemistry and Nitrogen Fractionation in the Mwc 480 Disk." The Astrophysical Journal 814 (1) (November 17): 53. doi:10.1088/0004-637x/814/1/53.

\section{Published Version}

doi:10.1088/0004-637x/814/1/53

\section{Permanent link}

http://nrs.harvard.edu/urn-3:HUL.InstRepos:24820077

\section{Terms of Use}

This article was downloaded from Harvard University's DASH repository, and is made available under the terms and conditions applicable to Open Access Policy Articles, as set forth at http:// nrs.harvard.edu/urn-3:HUL.InstRepos:dash.current.terms-of-use\#OAP

\section{Share Your Story}

The Harvard community has made this article openly available.

Please share how this access benefits you. Submit a story.

\section{Accessibility}


Draft Version November 12, 2015

Preprint typeset using $\mathrm{L}^{A} \mathrm{~T} \mathrm{E}$ X style emulateapj v. 05/12/14

\title{
CYANIDE PHOTOCHEMISTRY AND NITROGEN FRACTIONATION IN THE MWC 480 DISK
}

\author{
V.V. GuzMán ${ }^{1}$, K.I. ÖBerG ${ }^{1}$, R. LOOMis ${ }^{1}$, And C. QI ${ }^{1}$ \\ Draft version November 12, 2015
}

\begin{abstract}
$\mathrm{HCN}$ is a commonly observed molecule in Solar System bodies and in interstellar environments. Its abundance with respect to $\mathrm{CN}$ is a proposed tracer of UV exposure. HCN is also frequently used to probe the thermal history of objects, by measuring its degree of nitrogen fractionation. To address the utility of $\mathrm{HCN}$ as a probe of disks, we present ALMA observations of $\mathrm{CN}, \mathrm{HCN}, \mathrm{H}^{13} \mathrm{CN}$ and $\mathrm{HC}^{15} \mathrm{~N}$ toward the protoplanetary disk around Herbig Ae star MWC480, and of CN and HCN toward the disk around T Tauri star DM Tau. Emission from all molecules is clearly detected and spatially resolved, including the first detection of $\mathrm{HC}^{15} \mathrm{~N}$ in a disk. Toward MWC 480, CN emission extends radially more than 1" exterior to the observed cut-off of $\mathrm{HCN}$ emission. Quantitative modeling further reveals very different radial abundance profiles for $\mathrm{CN}$ and $\mathrm{HCN}$, with best-fit outer cut-off radii of $>300$ AU and $110 \pm 10$ AU, respectively. This result is in agreement with model predictions of efficient $\mathrm{HCN}$ photodissociation into $\mathrm{CN}$ in the outer-part of the disk where the vertical gas and dust column densities are low. No such difference in CN and HCN emission profiles are observed toward DM Tau, suggestive of different photochemical structures in Herbig Ae and T Tauri disks. We use the HCN isotopologue data toward the MWC 480 disk to provide the first measurement of the ${ }^{14} \mathrm{~N} /{ }^{15} \mathrm{~N}$ ratio in a disk. We find a low disk averaged ${ }^{14} \mathrm{~N} /{ }^{15} \mathrm{~N}$ ratio of $200 \pm 100$, comparable to what is observed in cloud cores and comets, demonstrating interstellar inheritance and/or efficient nitrogen fractionation in this disk.

Subject headings: astrochemistry, ISM: molecules, protoplanetary disks, radio lines; techniques: high angular resolution
\end{abstract}

\section{INTRODUCTION}

Protoplanetary disks are characterized by radial and vertical temperature and UV radiation gradients due to the exposure of disk surfaces to stellar and interstellar radiation, and attenuation of this radiation by dust and gas (Calvet et al. 1991; Herczeg et al. 2002). This radiation and temperature structure should result in a chemically stratified disk structure. Atoms and radicals (e.g. CN) alone are expected to survive in the UV-illuminated disk surface (Bergin et al. 2003). Molecules that are readily photo dissociated by UV radiation are mainly present in a protected layer between the disk surface and the cold midplane. In the midplane freeze-out of heavy atoms is expected to be nearly complete (Dutrey et al. 1997; Qi et al. 2013). The existence of these cold disk regions may be conductive to efficient fractionation of heavy isotopes into specific molecules as is introduced below.

Due to their different levels of photo resistance, $\mathrm{CN} / \mathrm{HCN}$ ratio has been proposed as a tracer of UV radiation fields in disks (Fuente et al. 1993). In addition to different vertical scale heights, Jonkheid et al. (2007) proposed that $\mathrm{CN}$ emission should be much more extended than HCN emission in disks around Herbig Ae stars due to UV penetration down to the midplane in the outer low-density disk. In the outer disk region, the disk molecular layer is effectively absent, resulting in a lack of HCN emission. Whether a similar structure should be expected around T Tauri stars is unclear because Herbig Ae stars are orders of magnitude more luminous than $\mathrm{T}$ Tauri stars. Oberg et al. (2011) used 3" Submillimeter

\footnotetext{
vguzman@cfa.harvard.edu

${ }^{1}$ Harvard-Smithsonian Center for Astrophysics, 60 Garden Street, Cambridge, MA 02138, USA
}

Array (SMA) observations of $\mathrm{CN}$ and $\mathrm{HCN}$ in a sample of disks to search for differences in the $\mathrm{CN}$ and $\mathrm{HCN}$ emission radii, and identified low-significance $1-2 \sigma$ differences in a handful of cases. Chapillon et al. (2012) observed $\mathrm{CN}$ and $\mathrm{HCN}$ emission at an angular-resolution of $1^{\prime \prime} .5-3^{\prime \prime}$ towards two T Tauri stars and one Herbig Ae star, but could not constrain the relative radial distribution because of different excitation characteristics of the observed $\mathrm{HCN}$ and $\mathrm{CN}$ lines.

$\mathrm{HCN}$ isotopologue ratios are potential probes of the disk thermal history. HCN is expected to become enriched in ${ }^{15} \mathrm{~N}$ (chemically fractionated) at low temperatures due to a small difference in zero-point energies for the ${ }^{14} \mathrm{~N}$ and ${ }^{15} \mathrm{~N}$ isotopologues (Terzieva \& Herbst 2000; Rodgers \& Charnlev 2008). Based on this scenario, the nitrogen isotopic compositions of different bodies in the Solar System (the Sun, rocky planets, gaseous planets, comets, meteorites, etc) have been used to understand their origin (see Mumma \& Charnlev 2011, and references therein). There are, however, other potential sources of ${ }^{15} \mathrm{~N}$ enrichment than chemical fractionation (Heays et al. 2014, and see Discussion). Measurements of nitrogen fractionation in analogs to the Solar Nebula are therefore key to interpreting Solar System isotopic data.

In this paper we present ALMA observations of $\mathrm{CN}$ and $\mathrm{HCN}$, and the $\mathrm{HCN}$ isotopologues $\mathrm{H}^{13} \mathrm{CN}$ and $\mathrm{HC}^{15} \mathrm{~N}$ towards the protoplanetary disks around $1.8 M_{\odot}$ Herbig Ae star MWC 480. We also present CN and HCN data toward the disk around $0.5 M_{\odot}$ T Tauri star DM Tau to enable a comparison between T Tauri and Herbig Ae photochemistry. Both stars are located in the Taurus star forming region at a distance of $140 \mathrm{pc}$, and are known to 
TABLE 1

OBSERVATION PARAMETERS

\begin{tabular}{|c|c|c|c|c|c|c|}
\hline Line & $\begin{array}{l}\text { Frequency } \\
\mathrm{GHz}\end{array}$ & $\begin{array}{c}E_{u} / k \\
\mathrm{~K}\end{array}$ & $\begin{array}{l}\text { Beam } \\
\operatorname{arcsec}\end{array}$ & $\underset{\circ}{\mathrm{PA}}$ & $\begin{array}{l}\text { Channel width } \\
\mathrm{km} \mathrm{s}^{-1}\end{array}$ & $\begin{array}{c}\text { Noise } \\
\text { mJy beam }^{-1} \text { channel }^{-1}\end{array}$ \\
\hline $\mathrm{CN} N=3-2, J=7 / 2-5 / 2$ & 340.24777 & 32.6 & $0.78 \times 0.45 / 0.65 \times 0.48$ & $-23.1 /-29.2$ & 0.1 & $12.3 / 9.2$ \\
\hline $\mathrm{HCN} J=4-3$ & 354.50548 & 42.5 & $0.77 \times 0.41 / 0.64 \times 0.40$ & $-24.1 /-29.0$ & 0.1 & $14.0 / 11.2$ \\
\hline $\mathrm{H}^{13} \mathrm{CN} J=3-2$ & 259.0118 & 24.9 & $0.74 \times 0.46$ & $-8.8^{\prime \prime}$ & 0.2 & 3.2 \\
\hline $\mathrm{HC}^{15} \mathrm{~N} J=3-2$ & 258.1571 & 24.8 & $0.74 \times 0.46$ & $-9.0^{\prime \prime}$ & 0.2 & 4.2 \\
\hline
\end{tabular}

Note: Two values are given for the beam, PA and noise, corresponding to the parameters in MWC 480 and DM Tau, respectively.
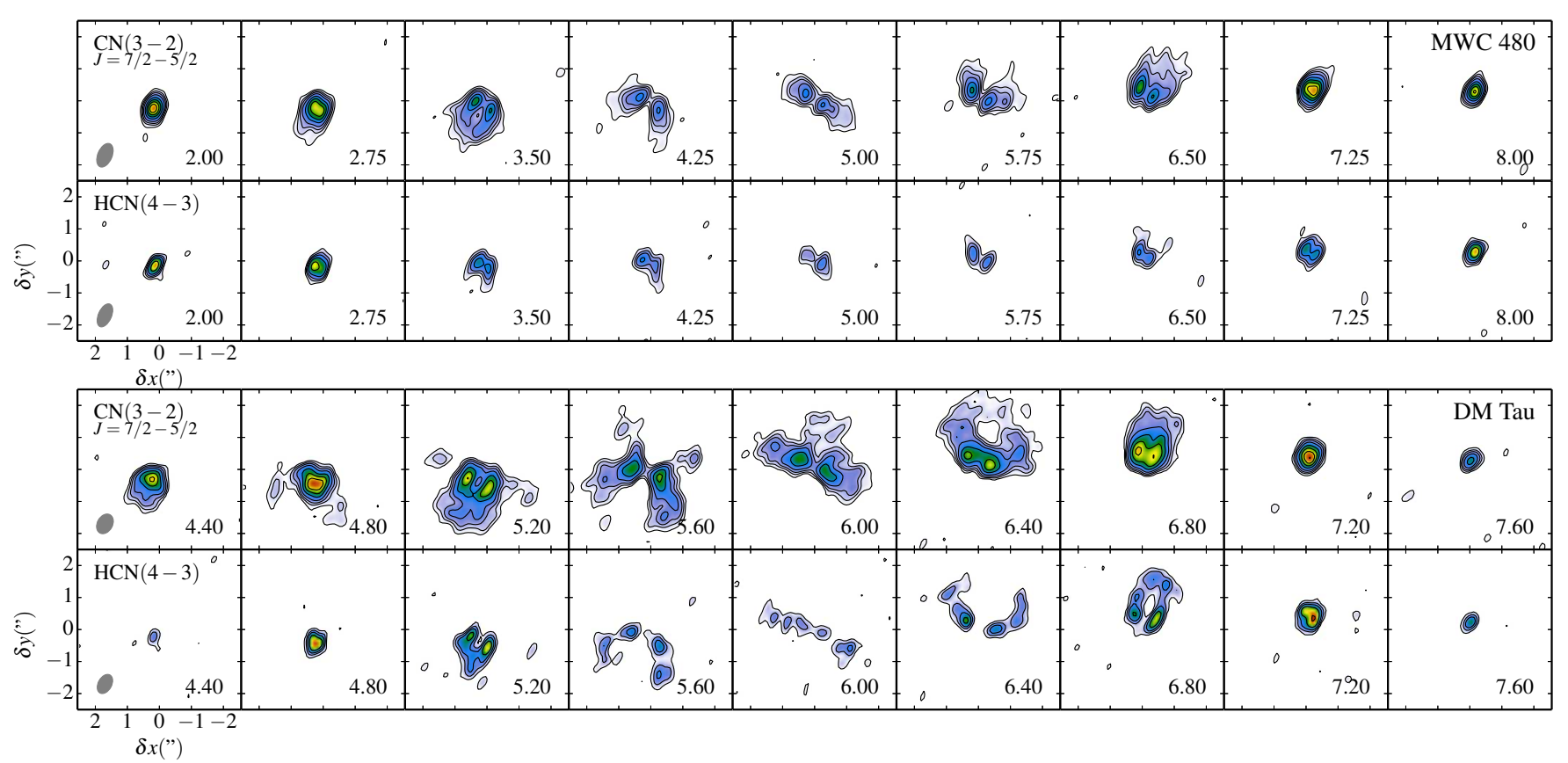

FIG. 1. - Channel maps of the HCN(4-3) and CN(3-2) line emission in MWC 480 (top) and DM Tau (bottom). The contour levels correspond to $3,5,7,10,15,20$ and $25 \sigma$ (Table 1). Synthesized beams are shown in the bottom left corner of the first panels.

present large, chemically rich disks (Dutrey et al. 1997; Henning et al. 2010; Oberg et al. 2010; Chapillon et al. 2012; Oberg et al. 2015). The observations are data reduction process are described in section 2. The observational results and analysis are presented in section 3 and a discussion is given in section 4. We summarize our findings an conclude in section 5.

\section{OBSERVATIONS AND DATA REDUCTION}

\section{1. $C N$ and $H C N$}

The HCN $J=4-3$ and CN $N=3-2, J=7 / 2-5 / 2$ lines were observed towards MWC 480 and DM Tau with the Atacama Large (sub-)Millimeter Array (ALMA) during Cycle 0 (PI: E. Chapillon, proposal 2011.0.00629). The Band 7 observations were carried out in October 2012 with 23 antennas. The total on-source observing time was $34 \mathrm{~min}$ and the baselines lengths ranged between 21 and $384 \mathrm{~m}$. The frequency bandpass was calibrated by observing the bright quasar J0423-013 at the begining of the observing run. The phase and amplitude temporal variations were calibrated by regularly observing the nearby quasars J0423-013, J01510-180 and Callisto. J01510-180 was used to derive the absolute flux scale. Two spectral windows of $468 \mathrm{MHz}$ bandwidth and $122 \mathrm{kHz}$ spectral resolution were centered on the CN and
HCN lines.

The data were calibrated by the ALMA staff using standard procedures, and retrieved from the public archive. We further self-calibrated the data in phase and amplitude in CASA, taking advantage of the bright continuum emission in both sources. The solutions of the self-calibration derived for the continuum were applied to each spectral window. The continuum was then subtracted from the visibilities using channels free of line emission to produce the spectral line cubes. The images were obtained by deconvolution of the visibilities using the CLEAN algorithm in CASA, with Briggs weighting and a robust parameter of 0.5 , resulting in an angular resolution of $0.6^{\prime \prime}$. A clean mask was created for each channel to select only regions with line emission during the cleaning process. Table 1 summarizes the observational parameters of the lines.

\section{2. $\mathrm{H}^{13} \mathrm{CN}$ and $\mathrm{HC}^{15} \mathrm{~N}$}

The $\mathrm{H}^{13} \mathrm{CN} J=3-2$ and $\mathrm{HC}^{15} \mathrm{~N} J=3-2$ lines were observed towards MWC 480 with ALMA during Cycle 2 as part of project 2013.1.00226 (PI: K.I. Öberg). These observations, partially described in Oberg et al. (2015), are part of a larger survey that did not include DM Tau. In brief, the Band 6 observations were carried out in June 

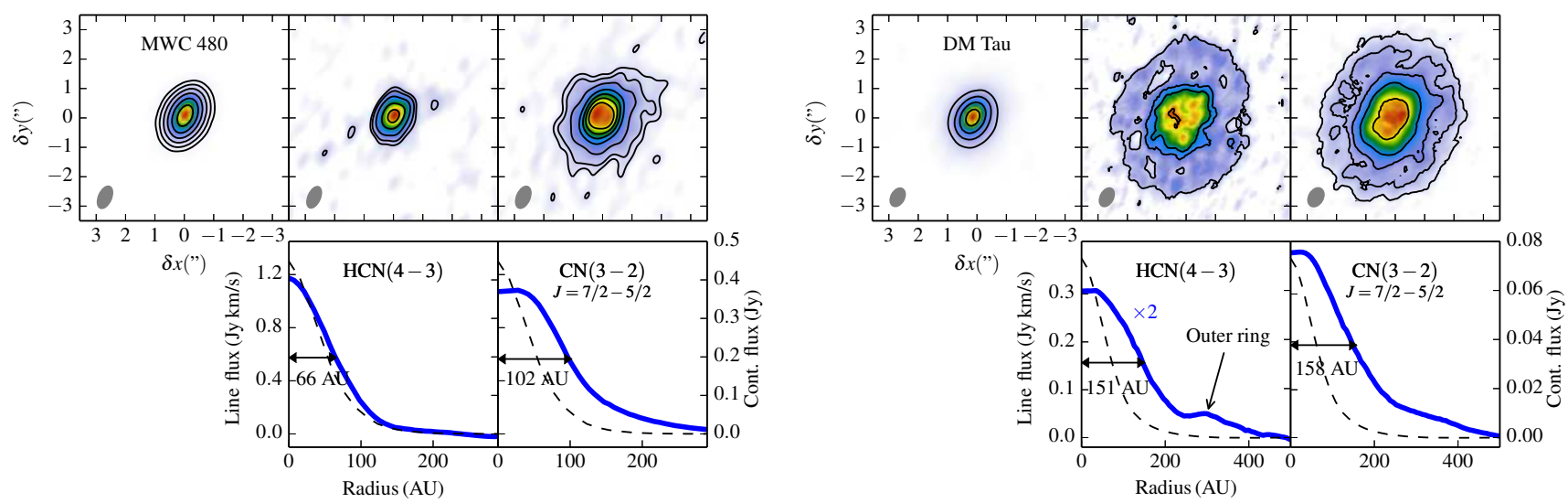

FIG. 2.- Dust continuum emission, moment zero maps (upper panels) and azimuthally-averaged emission profiles (bottom panels) of the HCN $J=4-3$ and CN $N=3-2, J=7 / 2-5 / 2$ lines (blue solid lines) in MWC480 (left) and DM Tau (right). The azimuthally-averaged dust continuum profiles are shown in dashed lines. The weaker HCN line in DM Tau has been multiplied by 2 to show the radial profile, and the outer ring near $300 \mathrm{AU}$, more clearly. Synthesized beams are shown in the bottom left panel corners. The double-arrows mark the half-light radii.

2014 with 33 antennas with baseline lengths spanning between 18 and $650 \mathrm{~m}$. The total on-source observing time was $24 \mathrm{~min}$. The quasar J0510+1800 was observed to calibrate the amplitude and phase temporal variations, as well as the frequency bandpass. The same quasar was used to derive the absolute flux scale, as no planet was available during the observations. Two spectral windows of $59 \mathrm{MHz}$ bandwidth and $61 \mathrm{kHz}$ channel spacing covered the $\mathrm{H}^{13} \mathrm{CN}$ and $\mathrm{HC}^{15} \mathrm{~N}$ lines.

A description of the phase and amplitude calibration, as well as the self-calibration, can be found in Oberg et al. (2015). The self-calibrated visibilities were cleaned in CASA using a robust parameter of 1.0, yielding an angular resolution of $\sim 0.6^{\prime \prime}$ (see Table 11). This selection of robustness parameter allowed us to improve the signal-to-noise ratio of the weak $\mathrm{H}^{13} \mathrm{CN}$ and $\mathrm{HC}^{15} \mathrm{~N}$ lines, while maintaining a good angular resolution. A clean mask was created for each channel, similarly to $\mathrm{CN}$ and $\mathrm{HCN}$, to select only regions with line emission during the cleaning process.

\section{OBSERVATIONAL RESULTS AND ANALYSIS \\ 3.1. Spatial distribution of $C N$ and $H C N$}

Figure 1 shows the channel maps of the CN $N=3-2$, $J=7 / 2-5 / 2$ and HCN $J=4-3$ lines in the MWC 480 and DM Tau disks. The velocity pattern characteristic of Keplerian rotation is seen for both lines and disks. Toward MWC 480, the HCN emission is clearly more compact than the CN emission in all channels. Such a difference in emission patterns is not observed toward the DM Tau disk. Furthermore, DM Tau exhibits a much more complex emission pattern in both the $\mathrm{CN}$ and $\mathrm{HCN}$ lines. The CN 'butterfly' shape in some of the central channels is caused by spectrally resolved hyperfine components. $\mathrm{HCN}$ seems to present an outer ring, whose potential origin is discussed in section 4

Fig. 2 shows the continuum, and velocity integrated maps and azimuthally averaged radial profiles of the $\mathrm{CN}$ and HCN lines towards MWC 480 and DM Tau. Toward MWC 480, these data representations confirm the noted differences in $\mathrm{CN}$ and $\mathrm{HCN}$ emission regions in Fig. 1] CN emission is detectable at a $2 \times$ larger radius
TABLE 2

AdOPTED PARAMETERS IN DISK MODEL FOR MWC 480.

\begin{tabular}{llll}
\hline \multicolumn{2}{c}{ Parameter } & Value & Ref. \\
\hline \multirow{2}{*}{ Scale height } & $H_{100}$ & $16 \mathrm{AU}$ & Guilloteau et al. (2011) \\
& $h$ & 1.25 & G \\
\hline \multirow{3}{*}{ Surface density } & $M_{\text {gas }}$ & $0.18 M_{\odot}$ & \\
& $\gamma$ & 0.75 & Guilloteau et al. (2011) \\
& $R_{c}$ & $81 \mathrm{AU}$ & \\
\hline \multirow{3}{*}{ Temperature } & $T_{100}$ & $23 \mathrm{~K}$ & Piétu et al. (2007) \\
& $q$ & 0.5 & Dartois et al. (2003) \\
& $\beta$ & 1.5 & \\
\hline
\end{tabular}

compared to HCN. The best-fit half-light radius for the $\mathrm{HCN}$ and $\mathrm{CN}$ emission toward MWC 480 are 66 and $102 \mathrm{AU}$, respectively. We further note that the $\mathrm{HCN}$ emission has a similar size to that of the dust continuum emission, which is shown in dashed-line in Fig. 2 In contrast, toward DM Tau the best-fit half-radius for $\mathrm{HCN}$ and $\mathrm{CN}$ are similar, 151 and 158 AU, respectively. The $\mathrm{HCN}$ outer ring hinted at in the DM Tau channel maps is also confirmed as a 'bump' around $300 \mathrm{AU}$ in the radial profile. No such ring is seen toward MWC 480.

To quantify the HCN and $\mathrm{CN}$ radial abundance profiles we modeled the $\mathrm{CN}$ and HCN emission using the procedure and disk density and temperature structure described in (Oberg et al. 2015). Key parameters adopted in the model are listed in Table 2. In brief, we model molecular abundances by defining them as power-laws $X=X_{0}\left(r / R_{0}\right)^{\alpha}$, with $X_{0}$ the abundance with respect to total hydrogen at $R_{0}=100 \mathrm{AU}$. We also included an outer cut-off radius, $R_{\text {out }}$. We ran grids of models for different outer radius $50<R_{\text {out }} / \mathrm{AU}<500$, power-law indexes $-2<\alpha<2$ and molecular abundances $10^{-14}<X_{100} / \mathrm{cm}^{-2}<10^{-9}$. The line emission was calculated using the radiative transfer code LIME (Brinch \& Hogerheijde 2010) assuming non-LTE excitation with collision rates from (Dumouchel et al. 2010) for HCN and from Lique et al. (2010) for CN. For the HCN isotopologues, we use the collisional rates of HCN. The model fitting was done in the $u-v$ plane by computing $\chi^{2}$, the weighted difference between model and observa- 

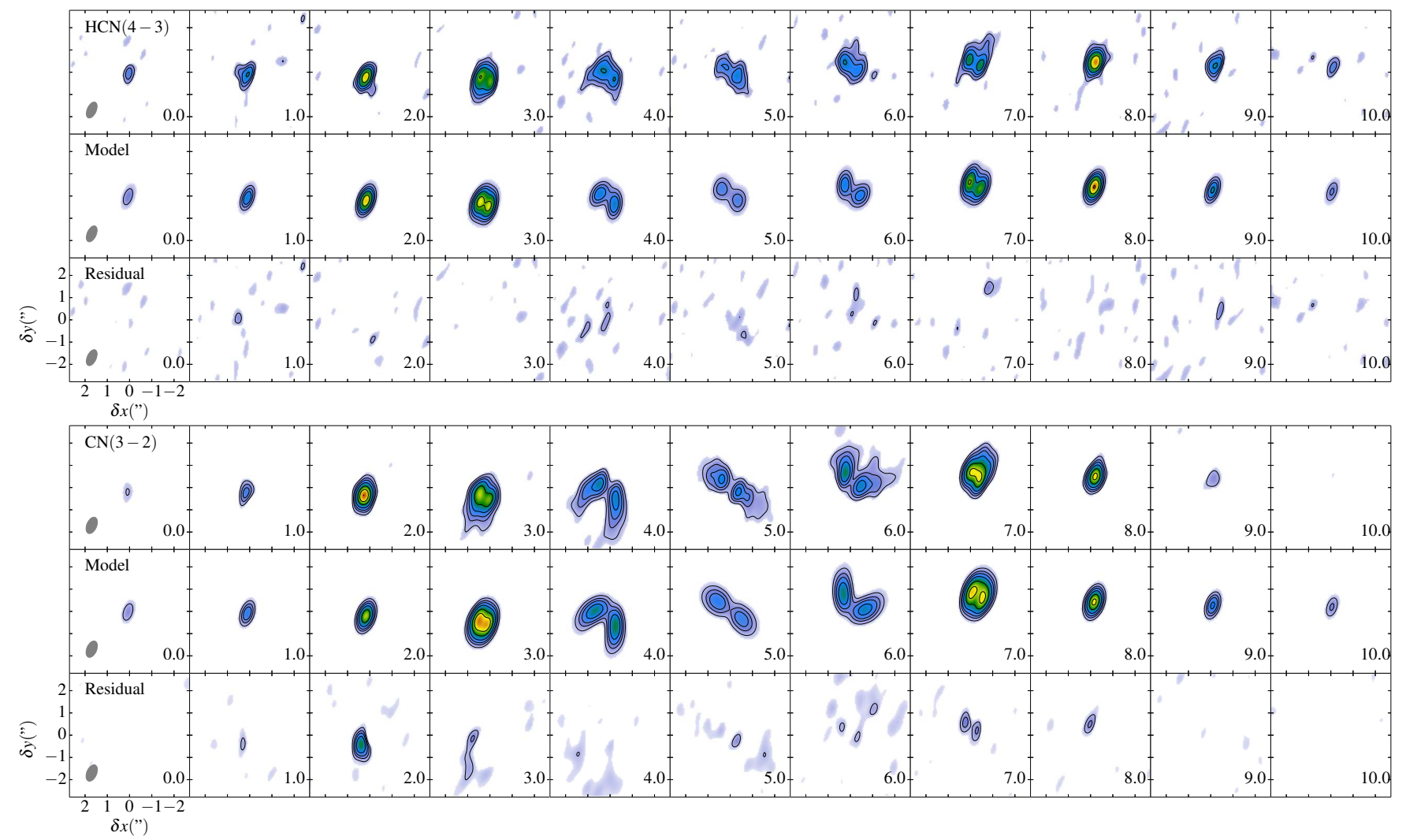

FIG. 3.- Channel maps of the observed CN and HCN emission in MWC 480 (upper panels), together with synthetic observations generated from the best-fit models for each line, corresponding to $X_{0}=1.0 \times 10^{-10}, R_{\text {out }}=350 \mathrm{AU}$ and $\alpha=-1$ for $\mathrm{CN}$, and $X_{0}=5.0 \times 10^{-12}$, $R_{\text {out }}=110 \mathrm{AU}$ and $\alpha=-1$ for $\mathrm{HCN}$ (middle panel). The residuals are shown in the bottom panels. The contour levels correspond to 3 , $5,7,10,15,20$ and $25 \sigma$.
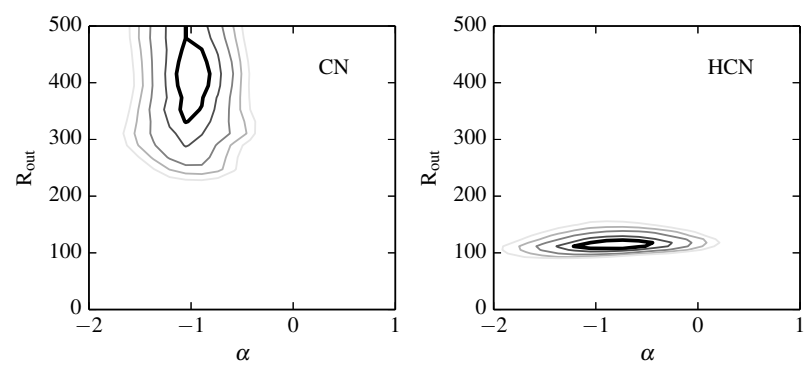

FIG. 4.- Contour plots of the $\chi^{2}$ surface for CN (left) and HCN (right) abundance profiles in the MWC 480 disk. The abundance at $100 \mathrm{AU}\left(X_{0}\right)$ is fixed to the best-fit model value (see Table 3 ). The thick contour corresponds to the $7 \sigma$ contour level, and the light-gray lines mark contours with $\Delta \chi^{2}=100,200,300$ and 400.

tions, for the real and imaginary parts of the complex visibilities. The best-fit models were obtained by minimizing $\chi^{2}$.

Fig. 4 shows the resulting contours of the $\Delta \chi^{2}=$ $\chi^{2}-\chi_{\min }^{2}$ distribution for the HCN and CN abundance profiles. The best fit model of both $\mathrm{CN}$ and $\mathrm{HCN}$ lines corresponds to a decreasing abundance profile as a function of radius, with $\alpha=-1$. The best-fit outer-radius of the HCN emission is $110 \mathrm{AU}$, while the outer-radius of the $\mathrm{CN}$ emission is constrained to $>300 \mathrm{AU}$, i.e. $>2 \times$ larger than HCN. The best-fit abundances at $100 \mathrm{AU}$ of $\mathrm{CN}$ and $\mathrm{HCN}$ are given in Table 3 but they are highly model dependent, i.e. they depend strongly on the assumed vertical abundance profile, and should not be di- rectly compared with model predictions. Table 3.2 lists the best fit parameters together with what are formally $7 \sigma$ error bars based on the data SNR. In our case the data SNR is high enough that we are dominated by stochastic noise from the radiative transfer code and the $7 \sigma$ error bar represents the deviation from the best fit model where there is a clear, visual misfit between model and data.

Figure 3 shows the excellent fit between our best-fit model and the data. Except for the low-velocity $\mathrm{CN}$ channels there are no significant residuals, and those residuals are due to the second hyperfine component of $\mathrm{CN}$, which is not included in our model. This hyperfine component is weak in MWC 480, but it is clearly seen in DM Tau (see Fig. 1). The good fit is also apparent when comparing the disk-integrated spectra (Fig. [5), where the spectra are extracted using a Keplerian mask.

\subsection{HCN isotopologues}

Figure 6 displays the moment-zero maps of the $\mathrm{H}^{13} \mathrm{CN}$ and $\mathrm{HC}^{15} \mathrm{~N}$ lines in MWC 480 together with the integrated emission of the blue and red shifted part of the the spectra, illustrating the disk rotation. The emission of the two isotopologues is compact, visually similar to the dust continuum and the HCN line emission.

We modeled the emission of the $\mathrm{HCN}$ isotopologues following the same procedure as for $\mathrm{CN}$ and $\mathrm{HCN}$ above. Figure 8 shows the $\Delta \chi^{2}$ distribution for the $\mathrm{H}^{13} \mathrm{CN}$ and $\mathrm{HC}^{15} \mathrm{~N}$ abundance profiles. The best fit parameters are listed in Table 3 . The good fit of the best-fit model to 

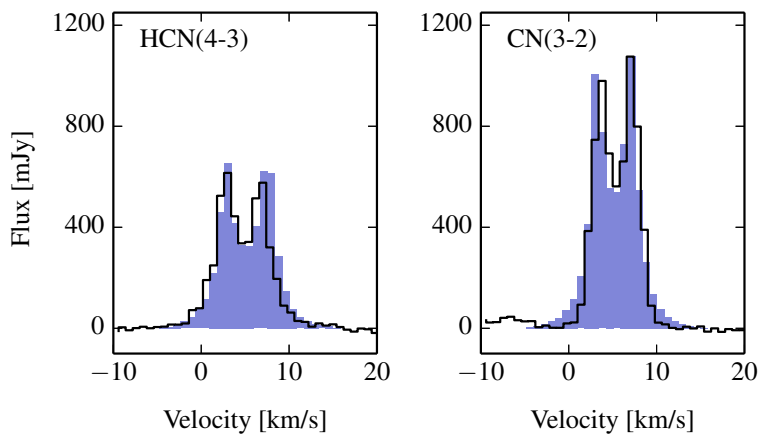

FIG. 5.- HCN and CN spectra integrated over the MWC 480 disk (black histograms), with the best-fit models overlaid on top (filled histograms).

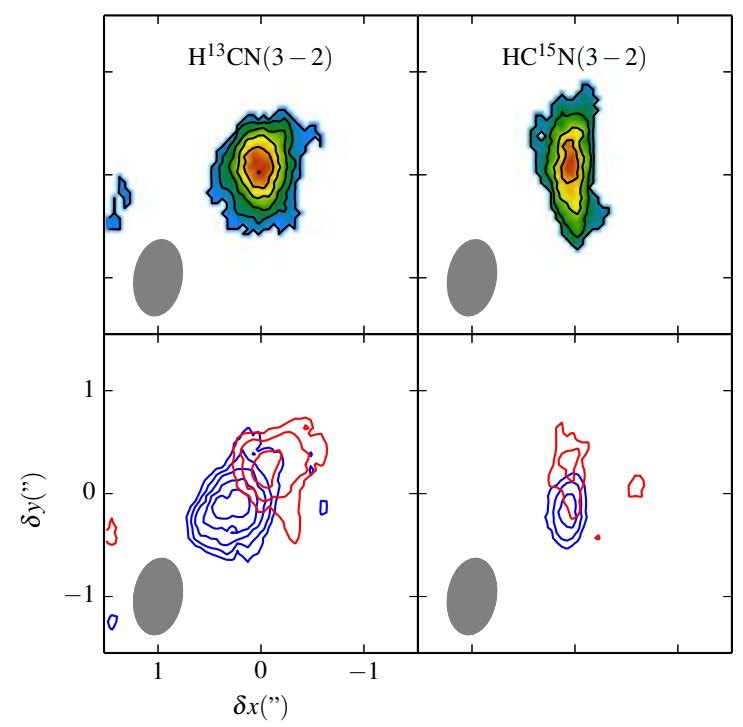

FIG. 6.- Moment zero maps (left panels) of the $\mathrm{H}^{13} \mathrm{CN} J=3-2$ and $\mathrm{HC}^{15} \mathrm{~N} J=3-2$ lines in the MWC 480 disk, shown together with integrated flux maps in two velocity bins around the $v_{l s r}$ (right panels), showing the velocity pattern.

the data disk integrated spectra (extracted using a Keplerian mask) is shown in Fig. 7 The best-fit model abundances at $100 \mathrm{AU}$ are given in Table 3. Similarly to $\mathrm{CN}$ and $\mathrm{HCN}$, the absolute abundances are uncertain, due to an unknown vertical abundance profile. Assuming that the $\mathrm{HCN}$ isotopologue emission originates in the same disk layer and that the lines are optically thin, their abundance ratio should be robust, however. This abundance ratio is $2.8 \pm 1.4$ and holds for the entire disk out to 100 AU since both lines are best fit by the same powerlaw index of -0.5 .

In the MWC 480 disk, the integrated $\mathrm{H}^{13} \mathrm{CN} / \mathrm{HCN}$ density flux ratio is $\sim 36$, which is lower than the ${ }^{12} \mathrm{C} /{ }^{13} \mathrm{C}$ isotopic ratios observed in the ISM, indicating that the $\mathrm{HCN}$ line is partially optically thick. We therefore use $\mathrm{H}^{13} \mathrm{CN}$ as a proxy of HCN to calculate the nitrogen fractionation in $\mathrm{HCN}$. Assuming an isotopic ratio of ${ }^{12} \mathrm{C} /{ }^{13} \mathrm{C}=70$, we derive a disk HCN isotopologue ratio $\mathrm{HC}^{14} \mathrm{~N} / \mathrm{HC}^{15} \mathrm{~N}=200 \pm 110$. To estimate this error we included a $30 \%$ uncertainty in the $\mathrm{C}$ isotopic ratio, as this value has been shown to vary both with time
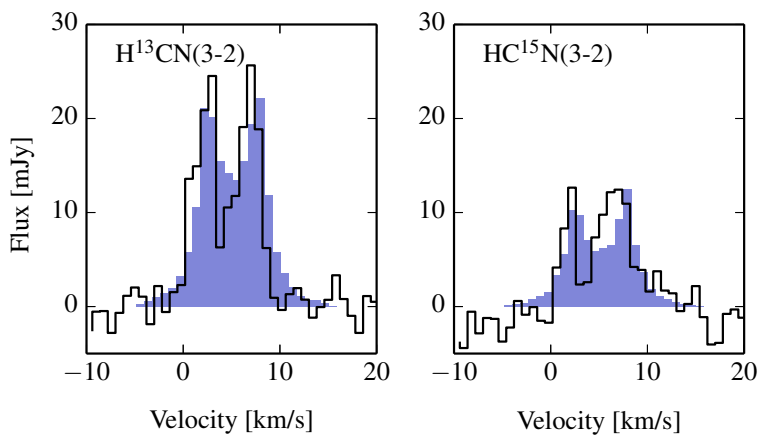

FIG. 7.- $\mathrm{H}^{13} \mathrm{CN}$ and $\mathrm{HC}^{15} \mathrm{~N}$ integrated spectra over the MWC 480 disk (black histograms) with the best-fit models overlaid on top (filled histograms).
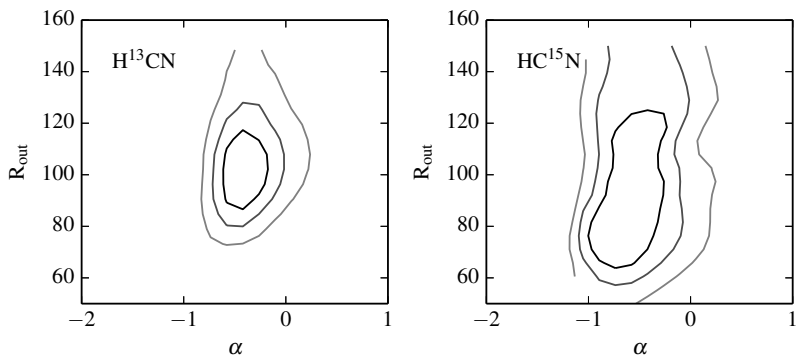

FIG. 8.- Contour plots of the $\chi^{2}$ surface for $\mathrm{H}^{13} \mathrm{CN}$ (left) and $\mathrm{HC}^{15} \mathrm{~N}$ (right) abundance profiles in the MWC 480 disk. The abundance at $100 \mathrm{AU}\left(X_{0}\right)$ is fixed to the best-fit model value (see Table 3). The three contours correspond to $1 \sigma, 2 \sigma$ and $3 \sigma$ confidence levels.

and density (Roueff et al. 2015). The main uncertainty $(\sim 85 \%)$, however, arises from the error in the measured $\mathrm{H}^{13} \mathrm{CN} / \mathrm{HC}^{15} \mathrm{~N}$ ratio.

\section{DISCUSSION}

\subsection{Photochemistry: $C N$ and $H C N$}

The observed $\mathrm{CN}$ and $\mathrm{HCN}$ disk emission profiles in the MWC 480 disk are well-fit by power-law abundance profiles with a radial cut-off that is two times larger for $\mathrm{CN}$ compared to $\mathrm{HCN}$. CN is thus significantly more radially extended than $\mathrm{HCN}$, i.e. we can exclude with a high level of confidence that excitation alone is responsible for the different $\mathrm{HCN}$ and $\mathrm{CN}$ emission profiles. This result is consistent with chemical model predictions of survival of $\mathrm{CN}$ and rapid photodissociation of HCN in the outer, low-density disk (Aikawa et al. 2002; Jonkheid et al. 2007). Indeed, these models predict that the $\mathrm{CN}$ abundance can remain constant and even increase with radius because radical formation is favored at low densities, and because CN is a major HCN photodissociation product. HCN, on the other hand, should only be present in the high-density parts of the disk where a shielded molecular layer exists.

The same models also predict a different vertical structure of $\mathrm{CN}$ and $\mathrm{HCN}$, with the $\mathrm{CN}$ abundance peaking close to the disk surface in the inner disk regions. Constraining the vertical abundances of these molecules requires multiple line transitions and/or direct observations of vertical emission patterns in edge-on disks. In the meantime, the derived abundance ratio of the two 
TABLE 3

Best-Fit PARAMETERS For THE MODELED ABUNDANCE PROFILES IN MWC 480.

\begin{tabular}{ccccc}
\hline & $\mathrm{CN}$ & $\mathrm{HCN}$ & $\mathrm{H}^{13} \mathrm{CN}$ & $\mathrm{HC}^{15} \mathrm{~N}$ \\
\hline$X_{0}$ & $(8.5 \pm 1.5) \times 10^{-11}$ & $(5.2 \pm 0.7) \times 10^{-12}$ & $(1.3 \pm 0.5) \times 10^{-13}$ & $(4.7 \pm 1.7) \times 10^{-14}$ \\
$\alpha$ & -1 & -1 & -0.5 & -0.5 \\
$R_{\text {out }[\mathrm{AU}]}$ & $>300$ & $110 \pm 10$ & $100 \pm 15$ & $95 \pm 30$ \\
\hline
\end{tabular}

Note: The absolute abundances are largely uncertain, due to the unknown vertical abundance and temperature profiles. However, because $\mathrm{H}^{13} \mathrm{CN}$ and $\mathrm{HC}^{15} \mathrm{~N}$ are expected to be, for the most part, co-spatial their abundance ratio does not suffer greatly from uncertainties in the vertical temperature profile. Therefore, the inferred $\mathrm{H}^{13} \mathrm{CN} / \mathrm{HC}^{15} \mathrm{~N}$ ratios is robust.

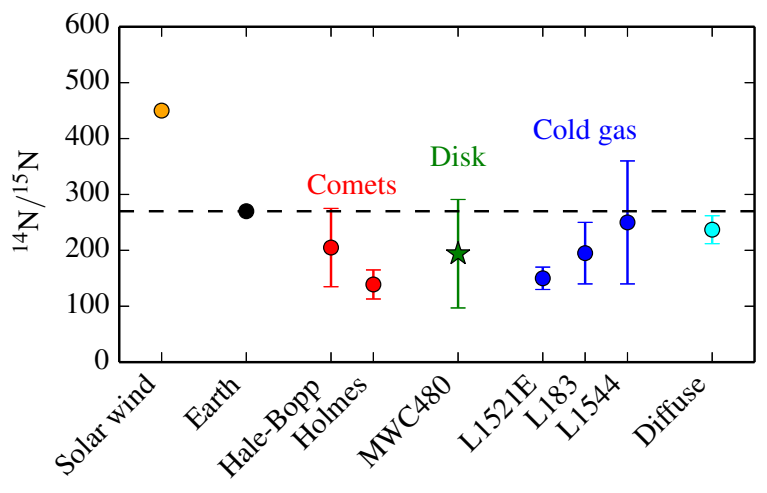

FIG. 9. ${ }^{14} \mathrm{~N} /{ }^{15} \mathrm{~N}$ isotopic ratios observed towards different Solar system bodies (Marty et al.|2011; Bockelée-Morvan et al.|2008) and in the ISM (Hily-Blant et al. 2013; Lucas \& Liszt 1998), and the disk around MWC 480.

molecules remains relatively unconstrained.

The emission of $\mathrm{CN}$ and $\mathrm{HCN}$ in the disk around $\mathrm{T}$ tauri star DM Tau exhibit different patterns compared to MWC 480. First, the spatial distribution (the extension of the emission) is similar for $\mathrm{CN}$ and $\mathrm{HCN}$ in the DM Tau disk. This difference between MWC 480 and DM Tau may be explained by the fact that the UV field around DM Tau should be substantially lower. Less vertical disk column may therefore be required to shield the outer disk regions, resulting in a shielded molecular layer out to many 100s of AU in the DM Tau disk. Second, HCN exhibits a low SNR outer ring around $300 \mathrm{AU}$, best identified in the azimuthally-averaged emission profile. The gap demarcating the main HCN disk from the outer ring is faintly seen in $\mathrm{CN}$ as well. This gap may be the result of micron-sized dust depletion at the same radius due to dust dynamics, which would enable UV radiation to penetrate down toward the midplane, photo-dissociating HCN. Such a dust lane has been observed toward TW Hya in scattered light (Debes et al. 2013). Similar high-quality scattered light observations toward DM Tau could resolve this question. If this scenario is confirmed, these ALMA observations suggest that $\mathrm{CN} / \mathrm{HCN}$ observations could be developed into a powerful probe of dust distributions and dynamics in the outer regions of disks.

\subsection{Nitrogen fractionation in the $M W C 480$ disk}

We used observations of HCN isotopologues to determine the level of nitrogen fractionation in the MWC 480 disk. Fig. 9 shows the measured ${ }^{14} \mathrm{~N} /{ }^{15} \mathrm{~N}$ ratio in the MWC 480 disk compared with different Solar system bodies and the ISM. Observations of the ${ }^{14} \mathrm{~N} /{ }^{15} \mathrm{~N}$ ratio in our Solar System reveal a clear difference between gaseous and rocky bodies Mumma \& Charnley 2011). A low $\mathrm{N}$ fractionation (i.e., high ${ }^{14} \mathrm{~N} /{ }^{15} \mathrm{~N}$ ) is observed toward the Sun and Jupiter (Marty et al. 2011), while a high fractionation (i.e., low ${ }^{14} \mathrm{~N} /{ }^{15} \mathrm{~N}$ ) is observed in the rocky planets, comets and meteorites (Bockelée-Morvan et al. 2008). Isotopic ratios in the coma of comets are determined remotely through high-resolution radio and optical spectroscopy. The ROSETTA space mission will hopefully provide $i n$-situ measurements of the ${ }^{14} \mathrm{~N} /{ }^{15} \mathrm{~N}$ ratio in the coma of comet $67 \mathrm{P} /$ Churyumov-Gerasimenko. Comets display the highest ${ }^{15} \mathrm{~N}$ enhancements among Solar System bodies (Mumma \& Charnley 2011). Prior to this study the only other place where such high nitrogen fractionation is found is cold pre-stellar cores (Hily-Blant et al. 2013; Wampfler et al. 2014). The cometary ${ }^{15} \mathrm{~N}$ abundance could thus signify an interstellar inheritance.

Interstellar inheritance is not the only possibility, however. Distinguishing between pre-Solar and Solar Nebula origins of nitrogen fractionation requires observations of nitrogen fractionation in protoplanetary disks. In general, HCN (isotopologue) formation begins with atomic nitrogen. At low temperatures ${ }^{15} \mathrm{~N}$ is preferentially incorporated into cyanides resulting in fractionation. Observations of HCN and HNC fractionation toward protostars present a tentative trend of the ${ }^{14} \mathrm{~N} /{ }^{15} \mathrm{~N}$ with temperature, supporting this scenario Wampfler et al. 2014). The second mechanism to produce an enhancement in ${ }^{15} \mathrm{~N}$ in $\mathrm{HCN}$ is isotope selective photodissociation of ${ }^{14} \mathrm{~N}^{15} \mathrm{~N}$ over ${ }^{14} \mathrm{~N}_{2}$, resulting in an enhanced atomic ${ }^{15} \mathrm{~N} /{ }^{14} \mathrm{~N}$ ratio. Theoretical models have shown that this mechanism can increase the ${ }^{14} \mathrm{~N} /{ }^{15} \mathrm{~N}$ ratio by up to a factor 10 in protoplanetary disks (Heavs et al. 2014).

Pre-stellar inheritance, low-temperature chemical fractionation in cold disk regions, and photochemically driven fractionation in disk atmospheres could thus all explain the observed nitrogen fractionation in comets. The presented HCN isotopologue observations in the MWC 480 disk cannot distinguish between these scenarios. Future, higher SNR and/or higher resolution disk studies could, however. The three scenarios should present very different radial and vertical ${ }^{14} \mathrm{~N} /{ }^{15} \mathrm{~N}$ profiles. Inheritance should produce an almost uniform ratio across the disk. Low-temperature chemical fractionation should result in a lower ${ }^{14} \mathrm{~N} /{ }^{15} \mathrm{~N}$ ratio in $\mathrm{HCN}$ in the outer, cold disk that is still protected from radiation and also a decreasing ratio toward the disk midplane. Finally, nitrogen fractionation from selective photodissociation should result in the lowest ${ }^{14} \mathrm{~N} /{ }^{15} \mathrm{~N}$ ratio in $\mathrm{HCN}$ closer to the disk atmosphere. In addition to detailed 
studies of individual sources, observations of the averaged ${ }^{14} \mathrm{~N} /{ }^{15} \mathrm{~N}$ ratio in disks exposed to different stellar radiation fields will also bring insight into the origin of $\mathrm{N}$ fractionation. If low-temperature chemical fractination is the dominant mechanism producing $\mathrm{HC}^{15} \mathrm{~N}$, then a multi-source study should result in a trend of increasing ${ }^{14} \mathrm{~N} /{ }^{15} \mathrm{~N}$ ratio with stellar radiation field. In contrast, the opposite behaviour should be observed if most of the $\mathrm{HC}^{15} \mathrm{~N}$ is produced by selective photodissociation in the surface layers. These observations will reveal how frequently protoplanetary disks are seeded with ${ }^{15} \mathrm{~N}$ enriched molecules, and whether additional enrichment occurs in the disk phase, changing the fractionation pattern during planet formation. The clear $\mathrm{HC}^{15} \mathrm{~N}$ detection in MWC 480 after 20 min integrated demonstrates that such observations will be readily achievable toward a sample of disks in a reasonable time frame.

\section{SUMMARY AND CONCLUSIONS}

Spatially and spectrally resolved ALMA observations toward the protoplanetary disk surrounding Herbig Ae star MWC 480 clearly show that its $\mathrm{CN}$ emission is $\sim 2$ times more extended than its HCN emission. We constructed a parametric model of the disk to obtain the underlying abundance profile of the two species and found that the observed difference in emission profiles correspond to similarly different abundance profiles for $\mathrm{CN}$ and HCN. This confirms theoretical model predictions which show that $\mathrm{HCN}$ is only abundant in disk regions where the radiation field has been attenuated sufficiently to prevent its photodissociation, while $\mathrm{CN}$ can survive in the outer low-density, unshielded disk regions. This difference in $\mathrm{CN}$ and $\mathrm{HCN}$ radial extent is not seen toward the disk around T Tauri star DM Tau, suggestive of different photochemical structures in disks around low and intermediate mass stars. The DM Tau disk also presents an intriguing outer ring in $\mathrm{HCN}$ (and potentially in $\mathrm{CN}$ ). Observation of $\mathrm{CN}$ and $\mathrm{HCN}$ coupled to detailed modeling clearly have great potential as tracers of UV radiation field strengths across protoplanetary disks.

We also presented the first detection of the $\mathrm{H}^{13} \mathrm{CN}$ and $\mathrm{HC}^{15} \mathrm{~N}$ isotopologues in a protoplanetary disk. We modeled the $\mathrm{H}^{13} \mathrm{CN}$ and $\mathrm{HC}^{15} \mathrm{~N}$ emission, and derive a disk average for the ${ }^{14} \mathrm{~N} /{ }^{15} \mathrm{~N}$ isotopic ratio of $200 \pm 100$ in MWC 480. This value, which is lower than the Solar one, is similar to what has been measured in comets and in the cold ISM. These observations show that with the current ALMA capabilities it is remarkable easy to detect the faint lines of rare istopologues of common molecules in protoplanetary disks making new detections in the near future very likely. Observations at higher sensitivity in MWC 480, as well as in other sources, should allow us to resolve variations of the ${ }^{14} \mathrm{~N} /{ }^{15} \mathrm{~N}$ ratio across the disk, and directly address the current fractionation puzzle of why different objects in our Solar system present different $\mathrm{N}$ enrichment levels.

\section{REFERENCES}

Aikawa, Y., van Zadelhoff, G. J., van Dishoeck, E. F., \& Herbst, E. 2002, A\&A, 386, 622

Bergin, E., Calvet, N., D’Alessio, P., \& Herczeg, G. J. 2003, ApJ, 591, L159

Bockelée-Morvan, D., Biver, N., Jehin, E., et al. 2008, ApJ, 679, L49

Brinch, C., \& Hogerheijde, M. R. 2010, A\&A, 523, A25

Calvet, N., Patino, A., Magris, G. C., \& D'Alessio, P. 1991, ApJ, 380,617

Calvet, N., D'Alessio, P., Watson, D. M., et al. 2005, ApJ, 630, L185

Chapillon, E., Guilloteau, S., Dutrey, A., Piétu, V., \& Guélin, M. 2012, A\&A, 537, A60

Chapillon, E., Dutrey, A., Guilloteau, S., et al. 2012, ApJ, 756, 58

Cleeves, L. I., Bergin, E. A., Alexander, C. M. O. '., et al. 2014, Science, 345, 1590

Dartois, E., Dutrey, A., \& Guilloteau, S. 2003, A\&A, 399, 773

Debes, J. H., Jang-Condell, H., Weinberger, A. J., Roberge, A., \& Schneider, G. 2013, ApJ, 771, 45

Dumouchel, F., Faure, A., \& Lique, F. 2010, MNRAS, 406, 2488

Dutrey, A., Guilloteau, S., \& Guelin, M. 1997, A\&A, 317, L55

Fuente, A., Martin-Pintado, J., Cernicharo, J., \& Bachiller, R. 1993, A\&A, 276, 473

Fuente, A., Rodrıguez-Franco, A., Garcia-Burillo, S., Martın-Pintado, J., \& Black, J. H. 2003, A\&A, 406, 899

Gerin, M., Marcelino, N., Biver, N., et al. 2009, A\&A, 498, L9

Guilloteau, S., Dutrey, A., Piétu, V., \& Boehler, Y. 2011, A\&A 529, A105

Heays, A. N., Visser, R., Gredel, R., et al. 2014, A\&A, 562, A61
Herczeg, G. J., Linsky, J. L., Valenti, J. A., Johns-Krull, C. M., \& Wood, B. E. 2002, ApJ, 572, 310

Henning, T., Semenov, D., Guilloteau, S., et al. 2010, ApJ, 714, 1511

Hily-Blant, P., Bonal, L., Faure, A., \& Quirico, E. 2013, Icarus, 223,582

Jonkheid, B., Dullemond, C. P., Hogerheijde, M. R., \& van

Dishoeck, E. F. 2007, A\&A, 463, 203

Lique, F., Spielfiedel, A., Feautrier, N., et al. 2010, J. Chem. Phys., 132, 024303

Lucas, R., \& Liszt, H. 1998, A\&A, 337, 246

Marty, B., Chaussidon, M., Wiens, R. C., Jurewicz, A. J. G., \& Burnett, D. S. 2011, Science, 332, 1533

Mumma, M. J., \& Charnley, S. B. 2011, ARA\&A, 49, 471

Öberg, K. I., Guzmán, V. V., Furuya, K., et al. 2015, Nature, 520,198

Öberg, K. I., Qi, C., Fogel, J. K. J., et al. 2010, ApJ, 720, 480

Öberg, K. I., Qi, C., Fogel, J. K. J., et al. 2011, ApJ, 734, 98

Piétu, V., Dutrey, A., \& Guilloteau, S. 2007, A\&A, 467, 163

Qi, C., Öberg, K. I., \& Wilner, D. J. 2013, ApJ, 765, 34

Rodgers, S. D., \& Charnley, S. B. 2008, ApJ, 689, 1448

Roueff, E., Loison, J. C., \& Hickson, K. M. 2015, A\&A, 576, A99

Simon, M., Dutrey, A., \& Guilloteau, S. 2000, ApJ, 545, 1034

Terzieva, R. \& Herbst, E. 2000, MNRAS, 317, 563

Wampfler, S. F., Jørgensen, J. K., Bizzarro, M., \& Bisschop, S. E. 2014, A\&A, 572, A24 